\title{
Boundedness of Rough Operators on Grand Variable Herz Spaces
}

\author{
Bechir Mahamat Acyl1,2, Shuangping Tao' , Omer Khalill1,3 \\ ${ }^{1}$ College of Mathematics and Statistics, Northwest Normal University, Lanzhou, China \\ ${ }^{2}$ Faculty of Sciences and Technology, Adam Barka University of Abeche, Abeche, Chad \\ ${ }^{3}$ Faculty of Education, Sudan University of Science and Technology, Khartoum, Khartoum State, Sudan \\ Email: bechirmahamatacyl@gmail.com, taosp@nwnu.edu.cn, us.omer2008@sustech.edu
}

How to cite this paper: Acyl, B.M., Tao, S. and Khalill, O. (2021) Boundedness of Rough Operators on Grand Variable Herz Spaces. Applied Mathematics, 12, 614-626. https://doi.org/10.4236/am.2021.127044

Received: April 22, 2021

Accepted: July 16, 2021

Published: July 19, 2021

Copyright () 2021 by author(s) and Scientific Research Publishing Inc. This work is licensed under the Creative Commons Attribution International License (CC BY 4.0).

http://creativecommons.org/licenses/by/4.0/

\begin{abstract}
In this paper, we prove the boundedness of Calderón-Zygmund singular integral operators $T_{\Omega}$ on grand Herz spaces with variable exponent under some conditions.

\section{Keywords}

Calderón-Zygmund Singular Integral Operator, Grand Herz Spaces, Variable Exponent
\end{abstract}

\section{Introduction}

Several years ago, the theory of function spaces with variable exponent has been extensively studied by some experts. initial work [1] by Kovóčik and Rákosník appearing in 1991. The Lebesgue spaces and some other function spaces have been studied in the variable exponent setting. Let $\Omega \in L^{s}\left(S^{n-1}\right)$ for $s>1$ be a homogeneous function of degree zero and satisfies

$$
\int_{S^{n-1}} \Omega\left(x^{\prime}\right) \mathrm{d} \sigma\left(x^{\prime}\right)=0,
$$

where $x^{\prime}=\frac{x}{|x|}$ for any $x \neq 0$. The Calderón-Zygmund singular integral operator $T_{\Omega}$ is defined by

$$
T_{\Omega} f(x)=\text { p.v. } \int_{\mathbb{R}^{n}} \frac{\Omega(x-y)}{|x-y|^{n}} f(y) \mathrm{d} y .
$$

This operator was firstly introduced by Calderón and Zygmund (see ([2] [3]) in which they proved that these operators are bounded on $L^{p}$, where $0<p<1$. They have proved the boundedness of Lebesgue spaces $L^{p}\left(\mathbb{R}^{n}\right)$ for all 
$1<p<\infty$. The boundedness is extended to the case on Herz spaces by Lu and Yang [4]. In [5], Lu, Ding and Yan proved that $T_{\Omega}$ and the commutator $\left[b, T_{\Omega}\right]$ are bounded on weighted $\left(L^{p}\left(\mathbb{R}^{n}\right), L^{q}\left(\mathbb{R}^{n}\right)\right)$. Recently, Humberto Rafeiro introduced Grand Lebesgue sequence spaces in [6], where various operators of harmonic analysis were studied in these spaces. In [7], Tan and Liu discussed some boundedness of homogeneous fractional integrals on variable exponent spaces. In [8], Humberto Rafeiro and Muhammad Asad Zaighum proposed grand variable Herz spaces $\dot{K}_{q}^{\alpha, p), \theta}\left(\mathbb{R}^{n}\right)$ and obtain the boundedness of sublinear operators on $\dot{K}_{q}^{\alpha, p), \theta}\left(\mathbb{R}^{n}\right)$.

Motivated by [8] our main purpose of this paper is to prove the boundedness of the Calderón-Zygmund singular integral operator $T_{\Omega}$ on grand Herz spaces with variable exponent. In Section 2, we first briefly recall some standard notations and lemmas in variable function spaces. Then will define the homogeneous and non-homogeneous Herz spaces with variable exponent and define the grand variable Herz space. In Section 3, the main result, we will prove the boundedness of Calderón-Zygmund singular integral operators on grand Herz spaces with variable exponent.

\section{Preliminaries and Lemmas}

Suppose $\Omega \subset \mathbb{R}^{n}$ and measurable function $p(\cdot): \Omega \rightarrow[1, \infty), L^{p(\cdot)}(\Omega)$ denotes the set of measurable functions $f$ on $\Omega$ such that for some $\lambda>0$,

$$
\int_{\Omega}\left(\frac{|f(x)|}{\lambda}\right)^{p(x)} \mathrm{d} x<\infty .
$$

This set becomes a Banach function space when equipped with the Luxemburg-Nakano norm

$$
\|f\|_{L^{(\cdot)}(\Omega)}=\inf \left\{\lambda>0: \int_{\Omega}\left(\frac{|f(x)|}{\lambda}\right)^{p(x)} \mathrm{d} x \leq 1\right\} .
$$

These spaces are referred to as variable $L^{p}$ spaces, since they generalize the standard $L^{p}$ spaces:

If $p(x)=p$ is constant, $L^{p(\cdot)}(\Omega)$ is isometrically isomorphic to $L^{p}(\Omega)$.

The space $L_{\text {loc }}^{p(\cdot)}(\Omega)$ is defined by

$$
L_{\text {loc }}^{p(\cdot)}(\Omega)=\left\{f: f \in L^{p(\cdot)}(E) \text { for all compact subsets } E \subset \Omega\right\} .
$$

Define $\mathcal{P}^{0}(E)$ to be the set of $p(\cdot): E \rightarrow(0, \infty)$ such that

$$
p^{-}=\operatorname{ess} \inf \{p(x): x \in E\}>0, p^{+}=\operatorname{ess} \sup \{p(x): x \in E\}<\infty .
$$

Define $\mathcal{P}(\Omega)$ to be the set of $p(\cdot): E \rightarrow[1, \infty)$ such that

$$
p^{-}=\operatorname{ess} \inf \{p(x): x \in \Omega\}>1, p^{+}=\operatorname{ess} \sup \{p(x): x \in \Omega\}<\infty .
$$

\subsection{Herz Space with Variable Exponent}

In this part, we present definitions of Herz spaces with variable exponent and 
use a notation in order to define those spaces. The important property for Herz spaces with variable exponents is the boundedness of the Hardy-Littlewood maximal operator.

Let $\imath \in \mathbb{Z}, \quad B_{l}:=\left\{x \in \mathbb{R}^{n}:|x| \leq 2^{\imath}\right\}, \quad B_{l}:=B_{l} \backslash B_{t-1}, \quad \chi_{i}:=\chi_{R_{i}}$.

$\mathbb{N}_{0}$ denotes the set of integers. For $m \in \mathbb{N}_{0}$, we denote $\tilde{\chi}_{m}:=\chi R_{m}$ if $m \geq 1$ and $\tilde{\chi}_{0}:=\chi B_{0}$.

Definition 2.1.1. (cf [9]). Suppose $\alpha \in \mathbb{R}, 0 \leq q \leq \infty$ and $p(\cdot): \mathbb{R}^{n} \rightarrow(0, \infty)$, $p(\cdot) \in \mathcal{P}\left(\mathbb{R}^{n}\right)$.

The homogeneous Herz space $\dot{K}_{p(\cdot)}^{\alpha, q}\left(\mathbb{R}^{n}\right)$ is defined by

$$
\dot{K}_{p(\cdot)}^{\alpha, q}\left(\mathbb{R}^{n}\right):=\left\{f \in L_{l o c}^{p(\cdot)}\left(\mathbb{R}^{n} \backslash 0\right):\|f\|_{\dot{K}_{p(\cdot)}^{\alpha, q}\left(\mathbb{R}^{n}\right)}<\infty\right\}
$$

where

$$
\|f\|_{K_{p(\cdot)}^{\alpha, q}\left(\mathbb{R}^{n}\right)}:=\left\|\left\{2^{\alpha l}\left\|f \chi_{l}\right\|_{L^{p(\cdot)}\left(\mathbb{R}^{n}\right)}\right\}_{l=-\infty}^{\infty}\right\|_{\ell^{q}(\mathbb{Z})} .
$$

The non-homogeneous Herz space $K_{p(\cdot)}^{\alpha, q}\left(\mathbb{R}^{n}\right)$ is

$$
K_{p(\cdot)}^{\alpha, q}\left(\mathbb{R}^{n}\right):=\left\{f \in L_{l o c}^{p(\cdot)}\left(\mathbb{R}^{n}\right):\|f\|_{K_{p(\cdot)}^{\alpha, q}\left(\mathbb{R}^{n}\right)}<\infty\right\},
$$

where

$$
\|f\|_{K_{P()}^{\alpha, q}\left(\mathbb{R}^{n}\right)}:=\left\|\left\{2^{\alpha m}\left\|f \tilde{\chi}_{m}\right\|_{L^{p()}\left(\mathbb{R}^{n}\right)}\right\}_{m=0}^{\infty}\right\|_{\ell^{q}\left(\mathbb{N}_{0}\right)} .
$$

Lemma 2.1.1. (cf [10]). If $p(\cdot) \in \mathcal{P}\left(\mathbb{R}^{n}\right)$ satisfying

$$
|p(x)-p(y)| \leq \frac{C}{-\log (|x-y|)},|x-y| \leq \frac{1}{2}
$$

and

$$
|p(x)-p(y)| \leq \frac{C}{\log (|x|+e)},|y| \geq|x|
$$

then $p(\cdot) \in \mathcal{B}\left(\mathbb{R}^{n}\right)$, that is, the Hardy-Littlewood maximal operator $M$ is bounded on $L^{p(\cdot)}\left(\mathbb{R}^{n}\right)$.

Lemma 2.1.2. ( $\operatorname{cf}[1])$. Suppose $p(\cdot) \in \mathcal{P}(\Omega)$, if $f \in L^{p(\cdot)}(\Omega)$ and $g \in L^{q^{\prime}(\cdot)}(\Omega)$, then $f g$ is integrable on $\Omega$ and

$$
\int|f(x) g(x)| \mathrm{d} x \leq r_{q}\|f\|_{L^{q(\cdot)}\left(R^{n}\right)}\|g\|_{L^{q^{\prime} \cdot(\cdot)}\left(\mathbb{R}^{n}\right)},
$$

where $r_{q}=1+1 / q_{-}-1 / p_{+}$.

Lemma 2.1.3. $(\operatorname{cf}[10])$. Suppose $p(\cdot) \in \mathcal{B}\left(\mathbb{R}^{n}\right)$. Then there exists a constant $C>0$ such that for all balls $B$ in $\mathbb{R}^{n}$,

$$
\frac{1}{|B|}\left\|\chi_{B}\right\|_{L^{q(\cdot)}\left(\mathbb{R}^{n}\right)}\left\|\chi_{B}\right\|_{L^{q^{(\cdot)}(\cdot)}\left(\mathbb{R}^{n}\right)} \leq C .
$$

Lemma 2.1.4. $(\operatorname{cf}[11])$. Define another variable exponent $\tilde{q}(\cdot)$ by $\frac{1}{p(x)}=\frac{1}{q}+\frac{1}{\tilde{q}(x)}\left(x \in \mathbb{R}^{n}\right)$. Then, we have 


$$
\|f g\|_{L^{p \cdot(\cdot)}\left(\mathbb{R}^{n}\right)} \leq C\|f\|_{L^{\tilde{q}(\cdot)}\left(\mathbb{R}^{n}\right)}\|g\|_{L^{q}\left(\mathbb{R}^{n}\right)},
$$

for all measurable functions $f$ and $g$.

Lemma 2.1.5. (cf [12]). Suppose $\Omega \in L^{s}\left(S^{n-1}\right), s \in[1, \infty]$. If $a>0, d \in(0, s]$ and $-n+\frac{(n-1) d}{s}<v<\infty$

$$
\left(\int_{|y| \leq a|x|}|y|^{v}|\Omega(x-y)|^{d} \mathrm{~d} y\right)^{1 / d} \lesssim\|\Omega\|_{L^{s}\left(s^{n-1}\right)}|x|^{(v+n) / d} .
$$

Lemma 2.1.6. (cf [13] Corollary 4.5.9.). Suppose $p \in \mathcal{P}^{\log }\left(\mathbb{R}^{n}\right)$. Then $\left\|\chi_{Q}\right\|_{p(\cdot)} \approx|Q|^{\frac{1}{p Q}}$ for any cube(or ball) $Q \subset \mathbb{R}^{n}$ where,

$$
\left\|\chi_{Q}\right\|_{p(\cdot)} \approx \begin{cases}|Q|^{\frac{1}{p(x)}} & \text { if }|Q| \leq 2^{n} \text { and } x \in Q \\ |Q|^{\frac{1}{p \infty}} & \text { if }|Q| \geq 1\end{cases}
$$

Lemma 2.1.7. $(\operatorname{cf}[8])$. Suppose $D>1$ and $q \in \mathcal{P}_{0, \infty}\left(\mathbb{R}^{n}\right)$. Then

$$
\frac{1}{c_{0}} r^{\frac{n}{q(0)}} \leq\left\|\chi_{R_{r, D r}}\right\|_{q(\cdot)} \leq c_{0} r^{\frac{n}{q(0)}}, \text { for } 0<r \leq 1,
$$

and

$$
\frac{1}{c_{\infty}} r^{\frac{n}{q_{\infty}}} \leq\left\|\chi_{R_{r, D r}}\right\|_{q(\cdot)} \leq c_{\infty} r^{\frac{n}{q_{\infty}}}, \text { for } r \geq 1
$$

respectively, where $c_{0} \geq 1$ and $c_{\infty} \geq 1$ depend on $D$, but do not depend on $r$.

Lemma 2.1.8. (cf [9]). Suppose $p(\cdot) \in \mathcal{B}\left(\mathbb{R}^{n}\right)$. Then there exists a positive constant $C$ such that for all balls $B$ in $\mathbb{R}^{n}$ and all measurable subsets $S \subset B$,

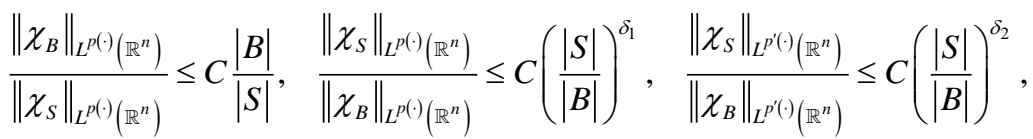

where $\delta_{1}, \delta_{2}$ are constants with $0<\delta_{1}, \delta_{2}<1$ and $\chi_{S}$ and $\chi_{B}$ are the characteristic functions of $S$ and $B$, respectively.

\subsection{Grand Space of Sequences}

Definition 2.2.1. (cf [6]). Let $1 \leq p<\infty$ and $\theta>0$, the grand Lebesgue sequence space is given by the norm

$$
\|\mathbf{x}\|_{\ell^{p, \theta}, \theta}:=\sup _{\varepsilon>0}\left(\varepsilon^{\theta} \sum_{k \in \mathbb{X}}\left|\chi_{k}\right|^{p(1+\varepsilon)}\right)^{\frac{1}{p(1+\varepsilon)}}=\sup _{\varepsilon>0} \varepsilon^{\frac{\theta}{p(1+\varepsilon)}}\|\mathbf{x}\|_{\ell^{p(1+\varepsilon)}}(\mathbb{X}) .
$$

where $\mathbf{x}=\left\{x_{k}\right\}_{k \in \mathbb{X}}$.

Note that the following nesting properties hold:

$$
l^{p(1-\varepsilon)} \hookrightarrow l^{p} \hookrightarrow l^{p), \theta_{1}} \hookrightarrow l^{p), \theta_{2}} \hookrightarrow l^{p(1+\delta)}
$$

for $0<\varepsilon<\frac{1}{p}, \delta>0$ and $0<\theta_{1} \leq \theta_{2}$. 


\subsection{Grand Variable Herz Spaces}

Definition 2.3.1. (cf [6]). Suppose $\alpha \in \mathbb{R}, 1 \leq p<\infty, q: \mathbb{R}^{n} \rightarrow[1, \infty), \theta>0$. We define the homogeneous grand variable Herz space by

$$
\dot{K}_{q(\cdot)}^{\alpha, p), \theta}\left(\mathbb{R}^{n}\right)=\left\{f \in L_{l o c}^{q(\cdot)}\left(\mathbb{R}^{n} \backslash\{0\}\right):\|f\|_{\dot{K}_{q(\cdot)}^{\alpha, p), \theta}\left(\mathbb{R}^{n}\right)}<\infty\right\},
$$

where

$$
\begin{aligned}
\|f\|_{\dot{K}_{q(\cdot)}^{\alpha, p), \theta}\left(\mathbb{R}^{n}\right)} & =\sup _{\varepsilon>0}\left(\varepsilon^{\theta} \sum_{k \in \mathbb{Z}} 2^{k \alpha p(1+\varepsilon)}\left\|f \chi_{k}\right\|_{L^{q(\cdot)}}^{p(1+\varepsilon)}\right)^{\frac{1}{p(1+\varepsilon)}} \\
& =\sup _{\varepsilon>0} \varepsilon^{\frac{1}{p(1+\varepsilon)}}\|f\|_{\dot{K}_{q(\cdot)}^{\alpha, p(1+\varepsilon)}\left(\mathbb{R}^{n}\right)} .
\end{aligned}
$$

In a similar way, non-homogeneous grand variable Herz spaces can be introduced.

\section{Main Results}

In the following theorem, we prove that Calderón-Zygmund singular integral operator $T_{\Omega}$ are bounded on grand Herz space with variable exponent.

Theorem 3.1. Let $1<p<\infty, q(\cdot) \in \mathcal{P}_{0, \infty}\left(\mathbb{R}^{n}\right)$ and $\Omega \in L^{s}\left(S^{n-1}\right)\left(s>q^{-}\right)$, $0<v \leq 1 \quad$ such that $\left[-n \delta_{1}-\left(v+\frac{n}{s}\right)-\frac{n}{q(0)}\right]<\alpha<\left[-n \delta_{1}-\left(v+\frac{n}{s}\right)+\frac{n}{q^{\prime}(0)}\right]$ and $\left[-n / q_{\infty}-n \delta_{1}-\left(v+\frac{n}{s}\right)\right]<\alpha<\left[n / q_{\infty}^{\prime}-n \delta_{1}-\left(v+\frac{n}{s}\right)\right]$. Let $T_{\Omega}$ bounded on $L^{q(\cdot)}\left(\mathbb{R}^{n}\right)$ satisfying the size condition (1.2). Then $T_{\Omega}$ is bounded on $\dot{K}_{q(\cdot)}^{\alpha, p, \theta}\left(\mathbb{R}^{n}\right)$.

Proof Theorem 3.1. Let $f \in \dot{K}_{q(\cdot)}^{\alpha, p), \theta}\left(\mathbb{R}^{n}\right)$.

Then, we obtain

$$
\begin{aligned}
\left\|T_{\Omega} f\right\|_{k_{q(\cdot)}^{\alpha, p), \theta}\left(\mathbb{R}^{n}\right)=} & \sup _{\varepsilon>0}\left(\varepsilon^{\theta} \sum_{k \in \mathbb{Z}} 2^{k \alpha p(1+\varepsilon)}\left\|\chi_{k} T_{\Omega} f\right\|_{L^{q(\cdot)}\left(\mathbb{R}^{n}\right)}^{p(1+\varepsilon)}\right)^{\frac{1}{p(1+\varepsilon)}} \\
\leq & \sup _{\varepsilon>0}\left(\varepsilon^{\theta} \sum_{k \in \mathbb{Z}} 2^{k \alpha p(1+\varepsilon)}\left(\sum_{l=-\infty}^{\infty}\left\|\chi_{k} T_{\Omega}\left(f \chi_{l}\right)\right\|_{L^{q(\cdot)}\left(\mathbb{R}^{n}\right)}^{p(1+\varepsilon)}\right)\right)^{\frac{1}{p(1+\varepsilon)}} \\
\leq & C \sup _{\varepsilon>0}\left(\varepsilon^{\theta} \sum_{k \in \mathbb{Z}} 2^{k \alpha p(1+\varepsilon)}\left(\sum_{l=-\infty}^{k-2}\left\|\chi_{k} T_{\Omega}\left(f \chi_{l}\right)\right\|_{L^{q(\cdot)}\left(\mathbb{R}^{n}\right)}\right)^{p(1+\varepsilon)}\right)^{\frac{1}{p(1+\varepsilon)}} \\
& +c \sup _{\varepsilon>0}\left(\varepsilon^{\theta} \sum_{k \in \mathbb{Z}} 2^{k \alpha p(1+\varepsilon)}\left(\sum_{l=k-1}^{k+1}\left\|\chi_{k} T_{\Omega}\left(f \chi_{l}\right)\right\|_{L^{q(\cdot)}\left(\mathbb{R}^{n}\right)}\right)^{p(1+\varepsilon)}\right)^{\frac{1}{p(1+\varepsilon)}} \\
& +c \sup _{\varepsilon>0}\left(\varepsilon^{\theta} \sum_{k \in \mathbb{Z}} 2^{k \alpha p(1+\varepsilon)}\left(\sum_{l=k+2}^{\infty}\left\|\chi_{k} T_{\Omega}\left(f \chi_{l}\right)\right\|_{L^{q(\cdot)}\left(\mathbb{R}^{n}\right)}\right)^{p(1+\varepsilon)}\right)^{\frac{1}{p(1+\varepsilon)}} \\
= & M_{1}+M_{2}+M_{3} .
\end{aligned}
$$


For $M_{2}$ using the $L^{q(\cdot)}\left(\mathbb{R}^{n}\right)$ boundedness of $T_{\Omega}$, we get

$$
\begin{aligned}
M_{2} & \leq c \sup _{\varepsilon>0}\left(\varepsilon^{\theta} \sum_{k \in \mathbb{Z}} 2^{k \alpha p(1+\varepsilon)}\left(\sum_{l=k-1}^{k+1}\left\|T\left(f \chi_{l}\right)\right\|_{L^{q(\cdot)}\left(\mathbb{R}^{n}\right)}\right)^{p(1+\varepsilon)}\right)^{\frac{1}{p(1+\varepsilon)}} \\
& \leq c \sup _{\varepsilon>0}\left(\varepsilon^{\theta} \sum_{k \in \mathbb{Z}} 2^{k \alpha p(1+\varepsilon)}\left(\sum_{l=k-1}^{k+1}\left\|f \chi_{l}\right\|_{L^{q(\cdot)}\left(\mathbb{R}^{n}\right)}\right)^{p(1+\varepsilon)}\right)^{\frac{1}{p(1+\varepsilon)}} \\
& \leq c \sup _{\varepsilon>0}\left(\varepsilon^{\theta} \sum_{k \in \mathbb{Z}} 2^{k \alpha p(1+\varepsilon)}\left\|f \chi_{k}\right\|_{L^{q(\cdot)}\left(\mathbb{R}^{n}\right)}^{p(1+\varepsilon)}\right)^{\frac{1}{p(1+\varepsilon)}} \\
& =c\|f\|_{\dot{K}_{q(\cdot)}^{\alpha, p), \theta}\left(\mathbb{R}^{n}\right)} .
\end{aligned}
$$

We estimate $M_{1}$, for each $k \in \mathbb{Z}$ and $l \leq k-2$ and a.e. $x \in B_{k}$ applying condition (1.2) and generalized Hölder's inequality, we have

$$
\begin{aligned}
\left|T_{\Omega}(f)(x)\right| & \leq c \int_{B_{l}} \frac{\Omega(x-y)}{|x-y|^{n}}|f(y)| \mathrm{d} y \\
& \leq c 2^{-k n} \int_{B_{l}}|\Omega(x-y)||f(y)| \mathrm{d} y \\
& \leq c 2^{-k n}\left\|\Omega(x-\cdot) \chi_{l}(\cdot)\right\|_{L^{q^{\prime}(\cdot)}\left(\mathbb{R}^{n}\right)}\|f\|_{L^{(\cdot)}\left(\mathbb{R}^{n}\right)} .
\end{aligned}
$$

Observation that $s>q^{\prime-}, \tilde{q}^{\prime}(\cdot)>1$ and $\frac{1}{q^{\prime}(x)}=\frac{1}{\tilde{q}^{\prime}(x)}+\frac{1}{s}$. Form lemmas 2.1.4 and 2.1.5, we get

$$
\begin{aligned}
& \left\|\Omega(x-\cdot) \chi_{l}(\cdot)\right\|_{L^{q^{(\cdot)}(\cdot)}\left(\mathbb{R}^{n}\right)} \leq\left\|\Omega(x-\cdot) \chi_{l}(\cdot)\right\|_{L^{s}\left(\mathbb{R}^{n}\right)}\left\|\chi_{l}(\cdot)\right\|_{L^{\tilde{q}^{(} \cdot(\cdot)}\left(\mathbb{R}^{n}\right)} \\
& \leq\left\|\Omega(x-\cdot) \chi_{l}(\cdot)\right\|_{L^{s}\left(\mathbb{R}^{n}\right)}\left\|\chi_{B_{l}}\right\|_{L^{\tilde{q}^{(\cdot)}}\left(\mathbb{R}^{n}\right)} \\
& \leq C 2^{-l v}\left(\int_{A_{l}}|\Omega(x-y)|^{s}|y|^{s v} \mathrm{~d} y\right)^{\frac{1}{s}}\left\|\chi_{B_{l}}\right\|_{L^{\tilde{q}(\cdot)}\left(\mathbb{R}^{n}\right)} \\
& \leq C 2^{-l v} 2^{k\left(v+\frac{n}{s}\right)}\|\Omega\|_{L^{5}\left(s^{n-1}\right)}\left\|\chi_{B_{1}}\right\|_{L^{q^{\prime}(\cdot)}\left(\mathbb{R}^{n}\right)} .
\end{aligned}
$$

When $\left|B_{l}\right| \leq 2^{n}$ and $x_{l} \in B_{l}$. From Lemma 2.1.6, we obtain

$$
\left\|\chi_{B_{l}}\right\|_{L_{q^{\prime}} \cdot(\cdot)\left(\mathbb{R}^{n}\right)} \approx\left|B_{l}\right|^{\frac{1}{\bar{q}^{(}\left(x_{l}\right)}} \approx\left\|\chi_{B_{l}}\right\|_{L^{q^{\prime}(\cdot)}\left(\mathbb{R}^{n}\right)}\left|B_{l}\right|^{-\frac{1}{s}} .
$$

When $\left|B_{l}\right| \geq 1$, we get

$$
\left\|\chi_{B_{l}}\right\|_{L^{\hat{q}^{\prime}(\cdot)}\left(\mathbb{R}^{n}\right)} \approx\left|B_{l}\right|^{\frac{1}{\vec{q}^{\prime}(\infty)}} \approx\left\|\chi_{B_{l}}\right\|_{L^{q^{\prime}(\cdot)}\left(\mathbb{R}^{n}\right)}\left|B_{l}\right|^{-\frac{1}{s}} .
$$

Consequently, we obtain

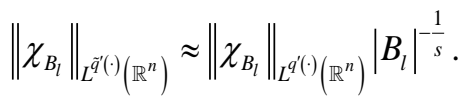

By Lemmas 2.1.3 and 2.1.8, we have

$$
\begin{aligned}
& \left\|T_{\Omega}(f) \chi_{I}\right\|_{L^{q(\cdot)}\left(\mathbb{R}^{n}\right)} \\
& \leq C 2^{-k n} 2^{-l v} 2^{k\left(v+\frac{n}{s}\right)}\|\Omega\|_{L^{s}\left(S^{n-1}\right)}\|f\|_{L^{q(\cdot)}\left(\mathbb{R}^{n}\right)}\left\|\chi_{B_{l}}\right\|_{L^{q^{(}(\cdot)}\left(\mathbb{R}^{n}\right)}\left\|\chi_{B_{k}}\right\|_{L^{q(\cdot)}\left(\mathbb{R}^{n}\right)}
\end{aligned}
$$




$$
\begin{aligned}
& \leq C 2^{-k n} 2^{-j v} 2^{k\left(v+\frac{n}{s}\right)}\|\Omega\|_{L^{s}\left(S^{n-1}\right)}\|f\|_{L^{q(\cdot)}\left(\mathbb{R}^{n}\right)}\left\|\chi_{B_{l}}\right\|_{L^{q^{\prime}(\cdot)}\left(\mathbb{R}^{n}\right)}\left|B_{l}\right|^{-\frac{1}{s}}\left\|\chi_{B_{k}}\right\|_{L^{q(\cdot)}\left(\mathbb{R}^{n}\right)} \\
& \leq C 2^{-k n+(k-l)\left(v+\frac{n}{s}\right)}\|\Omega\|_{L^{s}\left(S^{n-1}\right)}\|f\|_{L^{q(\cdot)}\left(\mathbb{R}^{n}\right)}\left\|\chi_{B_{l}}\right\|_{L^{q^{(\cdot)}(\cdot)}\left(\mathbb{R}^{n}\right)}\left\|\chi_{B_{k}}\right\|_{L^{Q^{(\cdot)}\left(\mathbb{R}^{n}\right)}} \leq C 2^{-k n+(k-l)\left(v+\frac{n}{s}\right)}\|\Omega\|_{L^{s}\left(S^{n-1}\right)}\|f\|_{L^{q(\cdot)}\left(\mathbb{R}^{n}\right)}\left\|\chi_{l}\right\|_{L^{q^{\prime}(\cdot)}\left(\mathbb{R}^{n}\right)}\left\|\chi_{k}\right\|_{L^{q(\cdot)}\left(\mathbb{R}^{n}\right)} \cdot
\end{aligned}
$$

From Lemma 2.1.7, we get

$$
2^{-k n}\left\|\chi_{k}\right\|_{L^{q(\cdot)}\left(\mathbb{R}^{n}\right)}\left\|\chi_{1}\right\|_{L^{q^{\prime}(\cdot)}\left(\mathbb{R}^{n}\right)} \leq c 2^{-k n} 2^{\left(\frac{k n}{q(0)}\right)} 2^{\left(\frac{l n}{q^{\prime}(0)}\right)} \leq c 2^{\frac{(l-k) n}{q^{\prime}(0)}} .
$$

Therefore,

$$
\begin{aligned}
& \left\|T_{\Omega}(f) \chi_{1}\right\|_{L^{q(\cdot)}\left(\mathbb{R}^{n}\right)} \\
& \leq c 2^{-k n} 2^{-l v} 2^{k\left(v+\frac{n}{s}\right)}\|\Omega\|_{L^{5}\left(s^{n-1}\right)}\|f\|_{L^{q(\cdot)}\left(\mathbb{R}^{n}\right)}\left\|\chi_{B_{l}}\right\|_{L^{q^{(}(\cdot)}\left(\mathbb{R}^{n}\right)}\left\|\chi_{B_{k}}\right\|_{L^{q(\cdot)}\left(\mathbb{R}^{n}\right)} \\
& \leq c 2^{(k-l)\left(v+\frac{n}{s}\right)} 2^{\frac{(l-k) n}{q^{\prime}(0)}}\|\Omega\|_{L^{s}\left(s^{n-1}\right)}\|f\|_{L^{q(\cdot)}\left(\mathbb{R}^{n}\right)} .
\end{aligned}
$$

Moreover, splitting $M_{1}$ by means of Minkowskis's inequality, we have

$$
\begin{aligned}
M_{1} \leq & c \sup _{\varepsilon>0}\left(\varepsilon^{\theta} \sum_{k=-\infty}^{-1} 2^{k \alpha p(1+\varepsilon)}\left(\sum_{l=-\infty}^{k-2}\left\|\chi_{k} T_{\Omega}\left(f \chi_{l}\right)\right\|_{L^{(\cdot)}\left(\mathbb{R}^{n}\right)}\right)^{p(1+\varepsilon)}\right)^{\frac{1}{p(1+\varepsilon)}} \\
& +c \sup _{\varepsilon>0}\left(\varepsilon^{\theta} \sum_{k=0}^{\infty} 2^{k \alpha p(1+\varepsilon)}\left(\sum_{l=-\infty}^{k-2}\left\|\chi_{k} T_{\Omega}\left(f \chi_{l}\right)\right\|_{L^{q(\cdot)}\left(\mathbb{R}^{n}\right)}\right)^{p(1+\varepsilon)}\right)^{\frac{1}{p(1+\varepsilon)}} \\
:= & M_{11}+M_{12} .
\end{aligned}
$$

For $M_{11}$ using (3.10) we get

$$
\begin{aligned}
& M_{11} \leq c \sup _{\varepsilon>0}\left(\varepsilon^{\theta} \sum_{k=-\infty}^{-1} 2^{k \alpha p(1+\varepsilon)}\left(\sum_{l=-\infty}^{k-2}\left\|\chi_{k}\right\|_{L^{q(\cdot)}\left(\mathbb{R}^{n}\right)} 2^{(k-l)\left(v+\frac{n}{s}\right)} 2^{\frac{(l-k) n}{q^{\prime}(0)}}\|\Omega\|_{L^{s}\left(S^{n-1}\right)}\left\|f \frac{\chi_{l}}{\chi_{l}}\right\|_{L^{q(\cdot)}\left(\mathbb{R}^{n}\right)}\right)^{p(1+\varepsilon)}\right)^{\frac{1}{p(1+\varepsilon)}} \\
& \leq c \sup _{\varepsilon>0}\left(\varepsilon^{\theta} \sum_{k=-\infty}^{-1} 2^{k \alpha p(1+\varepsilon)}\left(\sum_{l=-\infty}^{k-2}\left\|\frac{\chi_{k}}{\chi_{l}}\right\| 2^{(k-l)\left(v+\frac{n}{s}\right)} 2^{\frac{(l-k) n}{q^{\prime}(0)}}\|\Omega\|_{L^{5}\left(s^{n-1}\right)}\left\|f \chi_{l}\right\|_{L^{q(\cdot)}\left(\mathbb{R}^{n}\right)}\right)^{p(1+\varepsilon)}\right)^{\frac{1}{p(1+\varepsilon)}} \\
& \leq c \sup _{\varepsilon>0}\left(\varepsilon^{\theta} \sum_{k=-\infty}^{-1} 2^{\alpha l}\left(\sum_{l=-\infty}^{k-2} 2^{(k-l)\left[\alpha+n \delta_{1}+\left(v+\frac{n}{s}\right)-\frac{n}{q^{\prime}(0)}\right]}\|\Omega\|_{L^{5}\left(s^{n-1}\right)}\left\|f \chi_{l}\right\|_{L^{q(\cdot)}\left(\mathbb{R}^{n}\right)}\right)^{p(1+\varepsilon)}\right)^{\frac{1}{p(1+\varepsilon)}} \\
& \leq c\|\Omega\|_{L^{s}\left(S^{n-1}\right)} \sup _{\varepsilon>0}\left(\varepsilon^{\theta} \sum_{k=-\infty}^{-1}\left(\sum_{l=-\infty}^{k-2} 2^{\alpha l} 2^{(l-k)\left[-\alpha-n \delta_{1}-\left(v+\frac{n}{s}\right)+\frac{n}{q^{\prime}(0)}\right]}\left\|f \chi_{l}\right\|_{L^{(\cdot)}\left(\mathbb{R}^{n}\right)}\right)^{p(1+\varepsilon)}\right)^{\frac{1}{p(1+\varepsilon)}} \\
& \leq c\|\Omega\|_{L^{s}\left(S^{n-1}\right)} \sup _{\varepsilon>0}\left(\varepsilon^{\theta} \sum_{k=-\infty}^{-1}\left(\sum_{l=-\infty}^{k-2} 2^{\alpha l p(1+\omega)}\left\|f \chi_{l}\right\|_{L^{(\ell)}\left(\mathbb{R}^{n}\right)}^{p(1+\varepsilon)} 2^{(l-k) m p(1+\varepsilon)}\right)\right)^{\frac{1}{p(1+\varepsilon)}},
\end{aligned}
$$


where $m:=\left[-\alpha-n \delta_{1}-\left(v+\frac{n}{s}\right)+\frac{n}{q^{\prime}(0)}\right]>0$. Then we use hölder's inequality,

Fubini's theorem for series and $2^{-p(1+\varepsilon)}<2^{-p}$ to obtain

$$
\begin{aligned}
& M_{11} \leq c\|\Omega\|_{L^{s}\left(S^{n-1}\right)} \sup _{\varepsilon>0}\left(\varepsilon^{\theta} \sum_{k=-\infty}^{-1}\left(\sum_{l=-\infty}^{k-2} 2^{\alpha l p(1+\varepsilon)}\left\|f \chi_{l}\right\|_{L^{(\cdot)}\left(\mathbb{R}^{n}\right)}^{p(1+\varepsilon)} 2^{m p(1+\varepsilon)(l-k) / 2}\right)\right. \\
& \left.\times\left(\sum_{l=-\infty}^{k-2} 2^{m(p(1+\varepsilon))^{\prime}(l-k) / 2}\right)^{p(1+\varepsilon) /(p(1+\varepsilon))^{\prime}}\right)^{\frac{1}{p(1+\varepsilon)}} \\
& =c\|\Omega\|_{L^{s}\left(s^{n-1}\right)} \sup _{\varepsilon>0}\left(\varepsilon^{\theta} \sum_{k=-\infty}^{-1} \sum_{=-\infty}^{k-2} 2^{\alpha p(1+\varepsilon) l}\left\|f \chi_{k}\right\|_{L^{q(\cdot)}\left(\mathbb{R}^{n}\right)}^{p(1+\varepsilon)} 2^{m p(1+\varepsilon)(I-k) / 2}\right)^{\frac{1}{p(1+\varepsilon)}} \\
& =c\|\Omega\|_{L^{s}\left(S^{n-1}\right)} \sup _{\varepsilon>0}\left(\varepsilon^{\theta} \sum_{l=-\infty}^{-1} 2^{\alpha l p(1+\varepsilon)}\left\|f \chi_{k}\right\|_{L^{(!)}\left(\mathbb{R}^{n}\right)}^{p(1+\varepsilon)} \sum_{k=l+2}^{-1} 2^{m p(l-k) / 2}\right)^{\frac{1}{p(1+\varepsilon)}} \\
& \leq c\|\Omega\|_{L^{5}}\left(s^{n-1}\right) \sup _{\varepsilon>0}\left(\varepsilon^{\theta} \sum_{l=-\infty}^{-1} 2^{\alpha l p(1+\varepsilon)}\left\|f \chi_{k}\right\|_{L^{(\theta)}\left(\mathbb{R}^{n}\right)}^{p(1+\varepsilon)}\right)^{\frac{1}{p(1+\varepsilon)}} \\
& \leq c\|\Omega\|_{L^{s}\left(s^{n-1}\right)}\|f\|_{\dot{K}_{q(\cdot)}^{\alpha, p), \theta}\left(\mathbb{R}^{n}\right)} \cdot
\end{aligned}
$$

Now for $M_{12}$ using Minkowski's inequality, we have

$$
\begin{aligned}
M_{12} \leq & c \sup _{\varepsilon>0}\left(\varepsilon^{\theta} \sum_{k=0}^{\infty} 2^{k \alpha p(1+\varepsilon)}\left(\sum_{l=-\infty}^{-1}\left\|\chi_{k} T_{\Omega}\left(f \chi_{l}\right)\right\|_{L^{q(\cdot)}\left(\mathbb{R}^{n}\right)}\right)^{p(1+\varepsilon)}\right)^{\frac{1}{p(1+\varepsilon)}} \\
& +c \sup _{\varepsilon>0}\left(\varepsilon^{\theta} \sum_{k=0}^{\infty} 2^{k \alpha p(1+\varepsilon)}\left(\sum_{l=0}^{k-2}\left\|\chi_{k} T_{\Omega}\left(f \chi_{l}\right)\right\|_{L^{q(\cdot)}\left(\mathbb{R}^{n}\right)}\right)^{p(1+\varepsilon)}\right)^{\frac{1}{p(1+\varepsilon)}} \\
:= & B_{1}+B_{2} .
\end{aligned}
$$

The estimate for $B_{2}$ follows in similar manner to $M_{11}$ with $q^{\prime}(0)$ replaced by $q_{\infty}^{\prime}$ and using the fact that $\left[-\alpha-n \delta_{1}-\left(v+\frac{n}{s}\right)+n / q_{\infty}^{\prime}\right]>0 . \quad B_{1}$ using Lemma 2.1.7, we have

$$
2^{-k n}\left\|\chi_{k}\right\|_{L^{(\cdot)}\left(\mathbb{R}^{n}\right)}\left\|\chi_{l}\right\|_{L^{q^{(}(\cdot)}\left(\mathbb{R}^{n}\right)} \leq c 2^{-k n} 2^{\left(\frac{k n}{q_{\infty}}\right)} 2^{\left(\frac{l n}{q^{\prime}(0)}\right)} \leq c 2^{\left(\frac{-k n}{q^{\prime}(\infty)}\right)} 2^{\left(\frac{l n}{q^{\prime}(0)}\right)} .
$$

We get therefore,

$$
\begin{aligned}
\left\|T_{\Omega}(f) \chi_{1}\right\|_{L^{q(\cdot)}\left(\mathbb{R}^{n}\right)} & \leq c 2^{-k n} 2^{\left(\frac{k n}{q_{\infty}}\right)} 2^{\left(\frac{l n}{q^{\prime}(0)}\right)} 2^{(k-l)\left(v+\frac{n}{s}\right)}\|\Omega\|_{L^{s}\left(S^{n-1}\right)}\|f\|_{L^{q(\cdot)}\left(\mathbb{R}^{n}\right)} \\
& \leq c 2^{\left(\frac{-k n}{q^{\prime}(\infty)}\right)} 2^{\left(\frac{l n}{q^{\prime}(0)}\right)} 2^{(k-l)\left(v+\frac{n}{s}\right)}\|\Omega\|_{L^{s}\left(s^{n-1}\right)}\|f\|_{L^{q^{(\cdot)}\left(\mathbb{R}^{n}\right)}} .
\end{aligned}
$$

Now using (3.16) and fact that $\left[\alpha+n \delta_{1}+\left(v+\frac{n}{s}\right)-n / q_{\infty}^{\prime}\right]<0$ we have

$$
B_{1} \leq \sup _{\varepsilon>0}\left(\varepsilon^{\theta} \sum_{k=0}^{\infty} 2^{k \alpha p(1+\varepsilon)}\left(\sum_{l=-\infty}^{-1}\left\|\chi_{k} T_{\Omega} f\left(\chi_{l}\right)\right\|_{L^{q(\cdot)}\left(\mathbb{R}^{n}\right)}\right)^{p(1+\varepsilon)}\right)^{1 / p(1+\varepsilon)}
$$




$$
\begin{aligned}
& \leq \sup _{\varepsilon>0}\left(\varepsilon^{\theta} \sum_{k=0}^{\infty} 2^{k \alpha p(1+\varepsilon)}\left(\sum_{l=-\infty}^{-1}\left\|\chi_{k}\right\|_{L^{(q)}\left(\mathbb{R}^{n}\right)} 2^{\left(\frac{-k n}{q^{(}(\infty)}\right)} 2^{\left(\frac{l n}{q^{\prime}(0)}\right)} 2^{(k-l)\left(v+\frac{n}{s}\right)}\|\Omega\|_{L^{s}\left(S^{n-1}\right)}\|f\|_{L^{q(\cdot)}\left(\mathbb{R}^{n}\right)}\right)^{p(1+\varepsilon)}\right)^{1 / p(1+\varepsilon)} \\
& \leq c \sup _{\varepsilon>0}\left(\varepsilon^{\theta} \sum_{k=0}^{\infty} 2^{k \alpha p(1+\varepsilon)}\left(\sum_{l=-\infty}^{-1} 2^{n \delta_{1}(k-l)} 2^{\left(\frac{-k n}{q^{\prime}(\infty)}\right)} 2^{\left(\frac{l n}{q^{\prime}(0)}\right)} 2^{(k-l)\left(v+\frac{n}{s}\right)}\|\Omega\|_{L^{s}\left(s^{n-1}\right)}\left\|f \chi_{l}\right\|_{L^{q(\cdot)}\left(\mathbb{R}^{n}\right)}\right)^{p(1+\varepsilon)}\right)^{1 / p(1+\varepsilon)}
\end{aligned}
$$

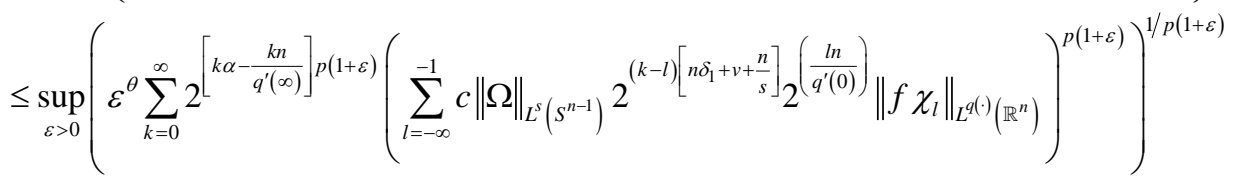

$$
\begin{aligned}
& \leq c\|\Omega\|_{L^{s}\left(S^{n-1}\right)} \sup _{\varepsilon>0}\left(\varepsilon^{\theta}\left(\sum_{l=-\infty}^{-1} 2^{(k-l)\left[n \delta_{1}+v+\frac{n}{s}\right]} 2_{2}\left(\frac{l n}{q^{\prime}(0)}\right)\left\|f \chi_{l}\right\|_{L^{q(\cdot)}\left(\mathbb{R}^{n}\right)}\right)^{p(1+\varepsilon)}\right)^{1 / p(1+\varepsilon)}
\end{aligned}
$$

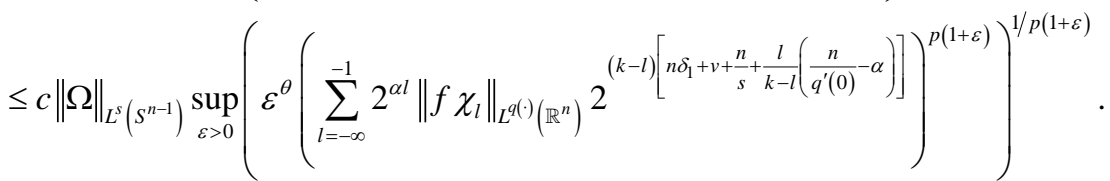

For applying Hölder's inequality and using the fact

$$
\begin{aligned}
& {\left[-\alpha-n \delta_{1}-\left(v+\frac{n}{s}\right)+\frac{n}{q^{\prime}(0)}\right]>0, \text { we get } } \\
& B_{1} \leq c \sup _{\varepsilon>0}\left(\varepsilon^{\theta}\left(\sum_{l=-\infty}^{-1} 2^{l \alpha p(1+\varepsilon)}\left\|f\left(\chi_{l}\right)\right\|_{L^{q(\cdot)}\left(\mathbb{R}^{n}\right)}^{p(1+\varepsilon)}\right)\right. \\
&\left.\times\left(\sum_{l=-\infty}^{-1} 2^{(k-l)\left[n \delta_{1}+v+\frac{n}{s}+\frac{l}{k-l}\left(\frac{n}{q^{\prime}(0)}-\alpha\right)\right](p(1+\varepsilon))^{\prime}}\right)^{p(1+\varepsilon) /(p(1+\varepsilon))^{\prime}}\right)^{1 / p(1+\varepsilon)} \\
& \leq c \sup _{\varepsilon>0}\left(\varepsilon^{\theta}\left(\sum_{l \in \mathbb{Z}} 2^{l \alpha p(1+\varepsilon)}\left\|f\left(\chi_{l}\right)\right\|_{L^{q(\cdot)}\left(\mathbb{R}^{n}\right)}^{p(1+\varepsilon)}\right)\right)^{1 / p(1+\varepsilon)} \\
& \leq c\|\Omega\|_{L^{s}\left(s^{n-1}\right)}\|f\|_{\dot{K}_{q(\cdot)}^{\alpha, p), \theta}\left(\mathbb{R}^{n}\right)} \cdot
\end{aligned}
$$

Next, we estimate $M_{3}$. For each $k \in \mathbb{Z}$ and $l \geq k+2$ and a.e $x \in B_{k}$; the size condition (3.10) and Hölder's inequality imply

$$
\left\|T_{\Omega}(f) \chi_{1}\right\|_{L^{(\cdot)}\left(\mathbb{R}^{n}\right)} \leq c 2^{-\ln +(k-l)\left(v+\frac{n}{s}\right)}\|\Omega\|_{L^{s}\left(S^{n-1}\right)}\|f\|_{L^{q(\cdot)}\left(\mathbb{R}^{n}\right)}\left\|\chi_{l}\right\|_{L^{q(\cdot)}\left(\mathbb{R}^{n}\right)}\left\|\chi_{k}\right\|_{L^{L^{(\cdot)}}\left(\mathbb{R}^{n}\right)}
$$

Similar to $M_{1}$, splitting $M_{3}$ by means of Minkowski's inequality, we have

$$
\begin{aligned}
M_{3} \leq & c \sup _{\varepsilon>0}\left(\varepsilon^{\theta} \sum_{k=-\infty}^{-1} 2^{k \alpha p(1+\varepsilon)}\left(\sum_{l=k+2}^{\infty}\left\|\chi_{k} T_{\Omega}\left(f \chi_{l}\right)\right\|_{L^{q(\cdot)}\left(\mathbb{R}^{n}\right)}\right)^{p(1+\varepsilon)}\right)^{\frac{1}{p(1+\varepsilon)}} \\
& +c \sup _{\varepsilon>0}\left(\varepsilon^{\theta} \sum_{k=0}^{\infty} 2^{k \alpha p(1+\varepsilon)}\left(\sum_{l=k+2}^{\infty}\left\|\chi_{k} T_{\Omega}\left(f \chi_{l}\right)\right\|_{L^{q(\cdot)}\left(\mathbb{R}^{n}\right)}\right)^{p(1+\varepsilon)}\right)^{\frac{1}{p(1+\varepsilon)}} \\
:= & M_{31}+M_{32} .
\end{aligned}
$$


For $M_{32}$ lemma 2.1 .7 yields

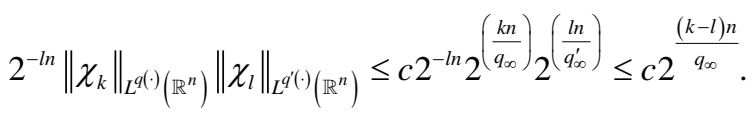

We get

$$
\begin{aligned}
\left\|T_{\Omega}(f) \chi_{l}\right\|_{L^{q(\cdot)}\left(\mathbb{R}^{n}\right)} & \leq c 2^{\delta_{1}(k-l)} 2^{\frac{(k-l) n}{q_{\infty}}} 2^{(k-l)\left(v+\frac{n}{s}\right)}\|\Omega\|_{L^{s}\left(S^{n-1}\right)}\left\|f \chi_{l}\right\|_{L^{q(\cdot)}\left(\mathbb{R}^{n}\right)} \\
& \leq c\|\Omega\|_{L^{s}\left(S^{n-1}\right)} 2^{(k-l)\left[\delta_{1}^{n+\frac{n}{q_{\infty}}+}+\left(v+\frac{n}{s}\right)\right]}\left\|f \chi_{l}\right\|_{L^{q(\cdot)}\left(\mathbb{R}^{n}\right)} .
\end{aligned}
$$

Using (3.22) for $M_{32}$, we have

$$
\begin{aligned}
& M_{32} \leq c \sup _{\varepsilon>0}\left(\varepsilon^{\theta} \sum_{k=0}^{\infty} 2^{k \alpha p(1+\varepsilon)}\left(\sum_{l=k+2}^{\infty}\|\Omega\|_{L^{s}\left(s^{n-1}\right)} 2^{(k-l)\left[\delta_{1} n+\frac{n}{q_{\infty}}+\left(v+\frac{n}{s}\right)\right]}\left\|f \chi_{l}\right\|_{L^{q(\cdot)}\left(\mathbb{R}^{n}\right)}\right)^{p(1+\varepsilon)}\right)^{\frac{1}{p(1+\varepsilon)}} \\
& \leq c\|\Omega\|_{L^{s}\left(S^{n-1}\right)} \sup _{\varepsilon>0}\left(\varepsilon^{\theta} \sum_{k=0}^{\infty}\left(\sum_{l=k+2}^{\infty} 2^{\alpha l}\left\|f \chi_{l}\right\|_{L^{q(\cdot)}\left(\mathbb{R}^{n}\right)} 2^{h(k-l)}\right)^{p(1+\varepsilon)}\right)^{\frac{1}{p(1+\varepsilon)}}, \\
& \text { where } h:=\left[\delta_{1} n+\frac{n}{q_{\infty}}+\left(v+\frac{n}{s}\right)+\alpha\right]>0 \text {. Then we use Hölder's inequality, Fubi- } \\
& \text { ni's theorem for series and } 2^{-p(1+\varepsilon)}<2^{-p} \text { to obtain }
\end{aligned}
$$

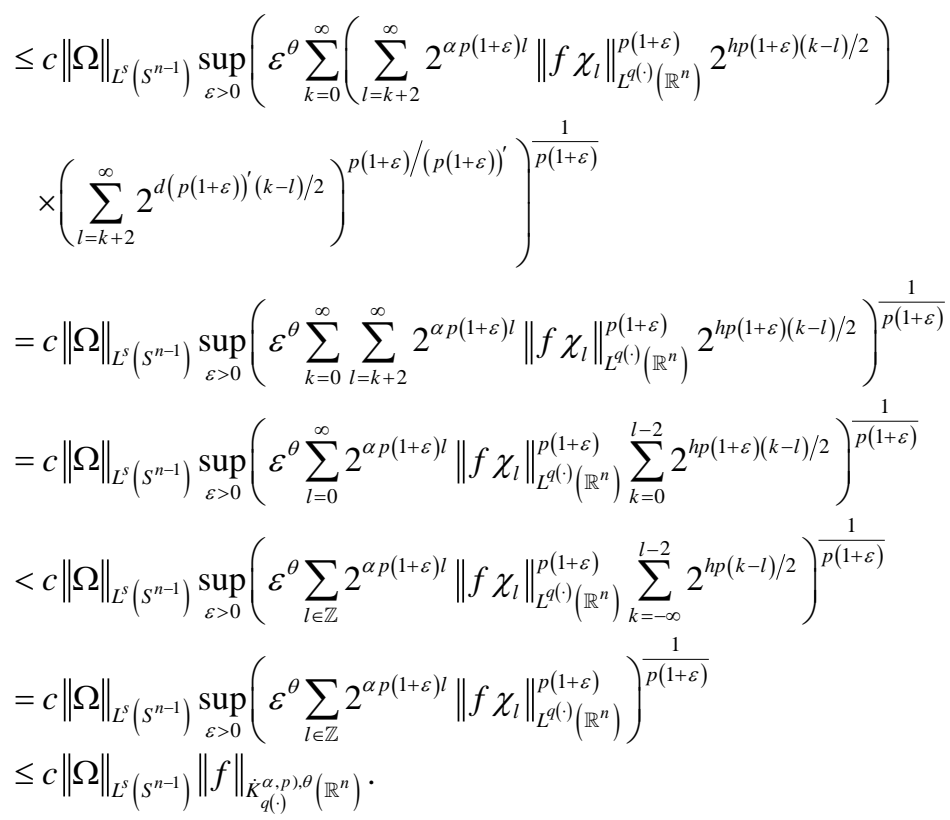

So for $M_{31}$ using Minkowski's inequality, we have

$$
\begin{aligned}
M_{31} \leq & c \sup _{\varepsilon>0}\left(\varepsilon^{\theta} \sum_{k=-\infty}^{-1} 2^{k \alpha p(1+\varepsilon)}\left(\sum_{l=k+2}^{-1}\left\|\chi_{k} T_{\Omega}\left(f \chi_{l}\right)\right\|_{L^{q(\cdot)}\left(\mathbb{R}^{n}\right)}\right)^{p(1+\varepsilon)}\right)^{\frac{1}{p(1+\varepsilon)}} \\
& +c \sup _{\varepsilon>0}\left(\varepsilon^{\theta} \sum_{k=-\infty}^{-1} 2^{k \alpha p(1+\varepsilon)}\left(\sum_{l=0}^{\infty}\left\|\chi_{k} T_{\Omega}\left(f \chi_{l}\right)\right\|_{L^{q(\cdot)}\left(\mathbb{R}^{n}\right)}\right)^{p(1+\varepsilon)}\right)^{\frac{1}{p(1+\varepsilon)}} \\
:= & V_{1}+V_{2} .
\end{aligned}
$$


The estimate for $V_{1}$ follows in a similar manner to $M_{32}$ with $q_{\infty}$ replaced by $q(0)$ and using the fact that $\left[\delta_{1} n+\frac{n}{q(0)}+\left(v+\frac{n}{s}\right)+\alpha\right]>0$. For $V_{2}$ using Lemma 2.1.7, we obtain

$$
\begin{aligned}
& 2^{-l n}\left\|\chi_{k}\right\|_{L^{q(\cdot)}\left(\mathbb{R}^{n}\right)}\left\|\chi_{l}\right\|_{L^{\prime}(\cdot)\left(\mathbb{R}^{n}\right)} \\
& \leq c 2^{-l n} 2^{\left(\frac{k n}{q(0)}\right)} 2^{\left(\frac{l n}{q_{\infty}^{\prime}}\right)} \leq c 2^{\left(\frac{k n}{q(0)}\right)} 2^{\left(\frac{-l n}{q_{\infty}}\right)} .
\end{aligned}
$$

By taking (3.26) and the fact that $\left[\delta_{1} n+\frac{n}{q(0)}+\left(v+\frac{n}{s}\right)+\alpha\right]>0$, we get

$$
\begin{aligned}
& V_{2} \leq \sup _{\varepsilon>0}\left(\varepsilon^{\theta} \sum_{k=-\infty}^{-1} 2^{k \alpha p(1+\varepsilon)}\left(\sum_{l=0}^{\infty}\left\|\chi_{k} T_{\Omega} f\left(\chi_{l}\right)\right\|_{L^{(\cdot)}\left(\mathbb{R}^{n}\right)}\right)^{p(1+\varepsilon)}\right)^{1 / p(1+\varepsilon)} \\
& \leq c \sup _{\varepsilon>0}\left(\varepsilon^{\theta} \sum_{k=-\infty}^{-1} 2^{k \alpha p(1+\varepsilon)}\left(\sum_{l=0}^{\infty} 2^{\delta_{1} n(k-l)} 2^{\frac{k n}{q(0)}} 2^{\frac{-l n}{q_{\infty}}} 2^{(k-l)\left(v+\frac{n}{s}\right)}\|\Omega\|_{L^{5}\left(S^{n-1}\right)}\left\|f\left(\chi_{l}\right)\right\|_{L^{q(\cdot)}\left(\mathbb{R}^{n}\right)}\right)^{p(1+\varepsilon)}\right)^{1 / p(1+\varepsilon)} \\
& \leq c\|\Omega\|_{L^{5}\left(s^{n-1}\right)} \sup _{\varepsilon>0}\left(\varepsilon^{\theta} \sum_{k=-\infty}^{-1} 2^{k \alpha p(1+\varepsilon)}\left(\sum_{l=0}^{\infty} 2^{(k-l)\left[\delta_{1} n+\left(v+\frac{n}{s}\right)\right]} 2^{\frac{k n}{q(0)}} 2^{\frac{-l n}{q_{\infty}}}\left\|f\left(\chi_{l}\right)\right\|_{L^{(\cdot)}\left(\mathbb{R}^{n}\right)}\right)^{p(1+\varepsilon)}\right)^{1 / p(1+\varepsilon)} \\
& \leq c\|\Omega\|_{L^{5}\left(s^{n-1}\right)} \sup _{\varepsilon>0}\left(\varepsilon^{\theta} \sum_{k=-\infty}^{-1} 2^{k\left(\alpha+\frac{n}{q(0)}\right) p(1+\varepsilon)}\left(\sum_{l=0}^{\infty} 2^{(k-l)\left[\delta_{1} n+\left(v+\frac{n}{s}\right)-\frac{l n}{q_{\infty}}\right]}\left\|f\left(\chi_{l}\right)\right\|_{L^{q(\cdot)}\left(\mathbb{R}^{n}\right)}\right)^{p(1+\varepsilon)}\right)^{1 / p(1+\varepsilon)} \\
& \leq c\|\Omega\|_{L^{s}\left(s^{n-1}\right)} \sup _{\varepsilon>0}\left(\varepsilon^{\theta}\left(\sum_{l=0}^{\infty} 2^{(k-l)\left[\delta_{1} n+\left(v+\frac{n}{s}-\frac{l n}{q_{\infty}}\right)\right]}\left\|f\left(\chi_{l}\right)\right\|_{L^{q(\cdot)}\left(\mathbb{R}^{n}\right)}\right)^{p(1+\varepsilon)}\right)^{1 / p(1+\varepsilon)} \\
& \leq c\|\Omega\|_{L^{s}\left(s^{n-1}\right)} \sup _{\varepsilon>0}\left(\varepsilon^{\theta} 2^{\alpha l}\left(\sum_{l=0}^{\infty} 2^{(k-l)\left[\delta_{1}^{n+(}\left(v+\frac{n}{s}\right)-l\left(\alpha+\frac{n}{q_{\infty}}\right)\right]}\left\|f\left(\chi_{l}\right)\right\|_{L^{q(\cdot)}\left(\mathbb{R}^{n}\right)}\right)^{p(1+\varepsilon)}\right)^{1 / p(1+\varepsilon)} .
\end{aligned}
$$

Finally by Hölder's inequality and $\left[\delta_{1} n+\left(v+\frac{n}{s}\right)+\left(\alpha+\frac{n}{q_{\infty}}\right)\right]>0$, we get

$$
\begin{aligned}
& V_{2} \leq c\|\Omega\|_{L^{s}\left(S^{n-1}\right)} \sup _{\varepsilon>0}\left(\varepsilon^{\theta}\left(\sum_{l=0}^{\infty} 2^{l \alpha p(1+\varepsilon)}\left\|f\left(\chi_{l}\right)\right\|_{L^{(l)}\left(\mathbb{R}^{n}\right)}^{p(1+\varepsilon)}\right)\right. \\
& \left.\times\left(\sum_{l=0}^{\infty} 2^{(k-l)\left[\delta_{1} n+\left(v+\frac{n}{s}\right)-l\left(\alpha+\frac{n}{q_{\infty}}(p(1+\varepsilon))^{\prime}\right)\right]}\right)^{p(1+\varepsilon) /(p(1+\varepsilon))^{\prime}}\right)^{1 / p(1+\varepsilon)} \\
& \leq c\|\Omega\|_{L^{s}\left(S^{n-1}\right)} \sup _{\varepsilon>0}\left(\varepsilon^{\theta} \sum_{l \in \mathbb{Z}} 2^{l \alpha p(1+\varepsilon)}\left\|f\left(\chi_{l}\right)\right\|_{L^{q(\cdot)}\left(\mathbb{R}^{n}\right)}^{p(1+\varepsilon)}\right)^{1 / p(1+\varepsilon)} \\
& \leq c\|\Omega\|_{L^{s}\left(S^{n-1}\right)}\|f\|_{\dot{K}_{q(\cdot)}^{\alpha, p), \theta}\left(\mathbb{R}^{n}\right)} .
\end{aligned}
$$


Combining the estimates for $M_{1}, M_{2}$ and $M_{3}$ yields

$$
\left\|T_{\Omega} f\right\|_{\dot{K}_{q(\cdot)}^{\alpha, p), \theta}\left(\mathbb{R}^{n}\right)} \leq c\|f\|_{\dot{K}_{q(\cdot)}^{\alpha, p,), \theta}\left(\mathbb{R}^{n}\right)} \cdot
$$

\section{Conclusion}

In this paper, we investigated the boundedness of rough operators on grand variable Herz space. We proved the boundedness of Calderón-Zygmund singular integral operators on grand Herz spaces with variable exponent under some conditions of variable exponent.

\section{Founding}

This work is supported by National Natural Science Foundation of China (61763044).

\section{Conflicts of Interest}

The authors declare no conflicts of interest regarding the publication of this paper.

\section{References}

[1] Kováčik, O. and Rákosník, J. (1991) On Spaces $L^{p(x)}$ and $W^{k, p(x)}$, Czechoslovak Mathematical Journal, 41, 592-618. https://doi.org/10.21136/CMJ.1991.102493

[2] Calderón, A.P. and Zygmund, A. (1952) On the Existence of Certain Singular Integrals. Acta Mathematica, 88, 85-139. https://doi.org/10.1007/BF02392130

[3] Calderón, A.P. and Zygmund, A. (1956) On Singular Integrals. American Journal of Mathematics, 78, 289-309. https://doi.org/10.2307/2372517

[4] Lu, S.Y. and Yang, D.C. (1997) The Continuity of Commutators on Herz-Type Spaces. The Michigan Mathematical Journal, 44, 255-281.

https://doi.org/10.1307/mmj/1029005703

[5] Shanzhen, L., Yong, D. and Dunyan, Y. (2007) Singular Integrals and Related Topics. World Scientific, Singapore.

[6] Rafeiro, H., Samko, S. and Umarkhadzhiev, S. (2018) Grand Lebesgue Sequence Spaces. Georgian Mathematical Journal, 25, 291-302.

https://doi.org/10.1515/gmj-2018-0017

[7] Jian, T.A. and Liu, Z.G. (2018) Some Boundedness of Homogeneous Fractional Integrals on Variable Exponent Function Spaces. ACTA Mathematics Science. Chinese Series, 58, 310-320.

[8] Hammad, N., Humberto, R. and Muhammad, A. (2020) A Note on the Boundedness of Sublinear Operators on Grand Variable Herz Spaces. Journal of Inequalities and Applications, 1, 1-13. https://doi.org/10.1186/s13660-019-2265-6

[9] Mitsuo, I. (2010) Boundedness of Sublinear Operators on Herz Spaces with Variable Exponent and Application to Wavelet Characterization. Analysis Mathematica, 36, 33-50. https://doi.org/10.1007/s10476-010-0102-8

[10] Wang, H. and Liao, F. (2020) Boundedness of Singular Integral Operators on Herz-Morrey Spaces with Variable Exponent. Chinese Annals of Mathematics, Series, 41, 99116. https://doi.org/10.1007/s11401-019-0188-7 
[11] Eiichi, N. and Yoshihiro, S. (2012) Hardy Spaces with Variable Exponents and Generalized Campanato Spaces. Journal of Functional Analysis, 262, 3665-3748. https://doi.org/10.1016/j.jfa.2012.01.004

[12] Muckenhoupt. B. and Wheeden, R.L. (1971) Weighted Norm Inequalities for Singular and Fractional Integrals. Transactions of the American Mathematical Society. 161, 249-258. https://doi.org/10.1090/S0002-9947-1971-0285938-7

[13] Diening, L., Harjulehto, P., Hst, P. and Ruzicka, M. (2011) Lebesgue and Sobolev Spaces with Variable Exponents. Springer, Berlin, Heidelberg.

https://doi.org/10.1007/978-3-642-18363-8 